\title{
Gemcitabine chemoresistance and molecular markers associated with gemcitabine transport and metabolism in human pancreatic cancer cells
}

\author{
Y Nakano', S Tanno*,2, K Koizumi', T Nishikawa', K Nakamura', M Minoguchi', T Izawa', Y Mizukami', \\ T Okumura ${ }^{2}$ and Y Kohgo'
}

'Third Department of Internal Medicine, Asahikawa Medical College, Asahikawa, Japan; ${ }^{2}$ Department of General Medicine, Asahikawa Medical College, Asahikawa, Japan

To identify predictive molecular markers for gemcitabine resistance, we investigated changes in the expression of four genes associated with gemcitabine transport and metabolism during the development of acquired gemcitabine resistance of pancreatic cancer cell lines. The expression levels of human equilibrative nucleoside transporter-I ( $h E N T I)$, deoxycytidine kinase (dCK), RRMI, and RRM2 mRNA were analysed by real-time light cycler-PCR in various subclones during the development of acquired resistance to gemcitabine. Real-time light cycler-PCR demonstrated that the expression levels of either RRM I or RRM2 progressively increased during the development of gemcitabine resistance. Expression of dCK was slightly increased in cells resistant to lower concentrations of gemcitabine, but was decreased below the undetectable level in higher concentration-resistant subclones. Expression of hENTI was increased in the development of gemcitabine resistance. As acquired resistance to gemcitabine seems to correlate with the balance of these four factors, we calculated the ratio of $h E N T I \times d C K J R R M I \times R R M 2$ gene expression in gemcitabine-resistant subclones. The ratio of gene expression decreased progressively with development of acquired resistance in gemcitabine-resistant subclones. Furthermore, the expression ratio significantly correlated with gemcitabine sensitivity in eight pancreatic cancer cell lines, whereas no single gene expression level correlated with the sensitivity. These results suggest that the sensitivity of pancreatic cancer cells to gemcitabine is determined by the ratio of four factors involved in gemcitabine transport and metabolism. The ratio of the four gene expression levels correlates with acquired gemcitabine-resistance in pancreatic cancer cells, and may be useful as a predictive marker for the efficacy of gemcitabine therapy in pancreatic cancer patients.

British Journal of Cancer (2007) 96, 457-463. doi:I0.1038/sj.bjc.6603559 www.bjcancer.com

Published online 16 January 2007

(C) 2007 Cancer Research UK

Keywords: pancreatic cancer; ribonucleotide reductase; deoxycytidine kinase; nucleoside transporter; gemcitabine resistance; predictive marker

Pancreatic adenocarcinoma is a common cancer with extremely poor prognosis. In the past few years, gemcitabine, a novel pyrimidine nucleoside analogue, has become the standard chemotherapeutic agent used in patients with pancreatic cancer (Rothenberg et al, 1996; Burris et al, 1997). However, even with this drug, most pancreatic adenocarcinomas exhibit an inexorable disease progression, and the clinical impact of gemcitabine remains modest owing to a high degree of inherent and acquired chemoresistance (Carmichael et al, 1996; Li et al, 2004). No clinical molecular markers have previously been shown that can predict a benefit from gemcitabine chemotherapy, and patients are treated empirically until disease progression or worsening therapy performance. Therefore, it is extremely important to determine

\footnotetext{
*Correspondence: Dr S Tanno;

E-mail: tanno I se@asahikawa-med.ac.jp

Revised 6 November 2006; accepted 22 November 2006; published online 16 January 2007
}

predictive molecular markers of gemcitabine resistance for more effective treatment of these tumours.

Gemcitabine has a complex pathway of metabolism, and there are many mechanisms that can contribute to gemcitabine cytotoxicity and/or chemoresistance (Habiro et al, 2004; Koizumi et al, 2005). In recent studies performed on human cancer cell lines, human equilibrative nucleoside transporter-1 (hENT1) was found to be the major gemcitabine transporter (Garcia-Manteiga et al, 2003). If gemcitabine is not transported into the cell via hENT1 it cannot inhibit cell growth (Mackey et al, 1998; Rauchwerger et al, 2000), but increased hENT1 abundance facilitates efficient cellular entry of gemcitabine and confers increased cytotoxicity (Mackey et al, 1999; Ritzel et al, 2001). Inside the cell, gemcitabine is phosphorylated by deoxycytidine kinase (dCK) in a rate-limiting step. Deficiency in dCK activity has been considered to be one of the main mechanisms responsible for the development of resistance to gemcitabine. Another factor in gemcitabine resistance is the overexpression of ribonucleotide reductase (RR). Ribonucleotide reductase is mainly responsible for 
the conversion of ribonucleosides to deoxyribonucleoside triphosphates (dNTPs), which are essential for DNA polymerisation and repair (Cory and Sato, 1983; Thelander and Berg, 1986; Fan et al, 1996; Zhou et al, 1998). RR consists of the dimerised large and small subunits, M1 and M2, respectively. The M1 subunit possesses a binding site for enzyme regulation (regulatory subunit), and the M2 subunit is involved with RR activity (catalytic subunit). Although it has been proposed that the genes for gemcitabine transport and metabolism are involved in the mechanism of cellular resistance to gemcitabine, it is not fully understood how gemcitabine influences its own transport and metabolism in the process of acquired resistance. Understanding alterations in expression of genes, which characterise the response of cancer cells to gemcitabine treatment in the process of acquired resistance, would allow us to improve therapeutic strategies for pancreatic cancer.

In this study, we established various gemcitabine-resistant subclones of human pancreatic cancer cell lines, and investigated changes in gene expression associated with gemcitabine transport and metabolism, that is, $h E N T 1, d C K, R R M 1$, and $R R M 2 \mathrm{mRNA}$, in the development of gemcitabine resistance. Quantitative RT-PCR analysis showed that the balance of the four gene expression levels is associated with inherent and acquired resistance to gemcitabine in pancreatic cancer cells. Resistance of cancer cells to gemcitabine is determined by the ratio of these gene expression levels, but not predicted by that of a single gene. The ratio of gene expression might be useful as a predictive marker for the efficacy of gemcitabine therapy in pancreatic cancer patients.

\section{MATERIALS AND METHODS}

\section{Chemicals}

Gemcitabine was a gift from Eli Lilly Pharmaceuticals (Indianapolis, IN, USA). All other chemicals were of analytical grade and commercially available.

\section{Cell culture and establishment of gemcitabine-resistant pancreatic cancer cells}

Eight human pancreatic adenocarcinoma cell lines, PK1, PCI43, KLM1, PK8, PK9, MIAPaCa2, KP1N, and BxPC3, were used in this study. PK1, KLM1, PK8, and PK9 cell lines were obtained from the Cell Resource Center for Biochemical Research (Tohoku University, Sendai, Japan). KP1N and MIAPaCa2 cell lines were purchased from the Health Science Research Resources Bank (Osaka, Japan). The PCI43 cell line was provided by Dr H Ishikura at Hokkaido University (Sapporo, Japan). PK1, PCI43, KLM1, PK8, PK9, KP1N, and BxPC3 were grown in RPMI 1640 media (Gibco, Paisley, Scotland) supplemented with $10 \%$ heat-inactivated fetal bovine serum in a humidified $5 \% \mathrm{CO}_{2}$ incubator at $37^{\circ} \mathrm{C}$. The MIAPaCa2 cell line was cultured in Dulbecco's modified Eagles medium (DMEM). Gemcitabine-resistant cells were generated by exposing the PCI43, PK1, and KLM1 cell lines to incrementally increasing gemcitabine concentrations starting at $3 \mathrm{nM}$. As the cells adapted to the drug, the gemcitabine concentration was doubled. The intermediate resistant variants were cultured for at least 4 weeks. The cell lines were named as follows: $G$ for gemcitabine, followed by the nM concentration at which the cell line grew logarithmically. The most resistant variants were PCI43-G4000, PK1-G4000, and KLM1-G4000 and were resistant to continuous exposure to gemcitabine at $4000 \mathrm{nM}$. Experiments were performed using cells in the exponential phase of growth.

\section{Drug cytotoxicity assay}

The relative cytotoxicity of gemcitabine in each cell line was assessed with a WST-1 assay using a Cell Counting Kit (Dojindo
Laboratories, Kumamoto, Japan). This assay is based on the reduction of a tetrazolium compound to a soluble derivative by the dehydrogenase enzymes of metabolically active cells. The absorbance $(450 \mathrm{~nm})$ is directly proportional to the number of living cells in culture. Cells were added to 96-well tissue culture plates $\left(3 \times 10^{3}\right.$ cells/well $)$ overnight and exposed to increasing concentrations $\left(10^{-2} 10^{3} \mu \mathrm{m}\right)$ of gemcitabine for $72 \mathrm{~h}$, after which the number of remaining living cells was determined according to the manufacturer's instructions. Chemosensitivity was expressed as the drug concentration that inhibited cell proliferation by $50 \%$ ( $\mathrm{IC}_{50}$ values) and was determined from concentration-effect relationship.

\section{Quantitative LightCycler RT - PCR}

Total RNA was extracted from each cell line and gemcitabineresistant subclones using the RNeasy Protect Mini Kit (Qiagen, Hilden, Germany) with the RNase-Free DNase Set (Qiagen) according to the manufacturer's instructions. Complementary DNA (cDNA) was produced from $1 \mu \mathrm{g}$ of RNA using an Oligo $(\mathrm{dT})_{12-18}$ Primer (Invitrogen, Carlsbad, CA, USA) and MMLV reverse transcriptase (Promega, Madison, WI, USA). Primers for hENT1, dCK, RRM1, RRM2, and glyceraldehyde-3-phosphate dehydrogenase (GAPDH) were based on the sequence of each gene (Entrez-PubMed) and designed by the program Primer 3. Oligonucleotides used as PCR primers are summarised in Table 1. Quantitative RT-PCR was performed in a LightCycler system (Roche Molecular Biochemicals, Mannheim, Germany) using SYBR Green fluorescence. In this system, all reactions were run in glass capillaries with a total volume of $20 \mu \mathrm{l}$. The reaction mixture consisted of $2 \mu \mathrm{l}$ of FastStart DNA Master SYBR Green I, SYBR Green I dye, and $10 \mathrm{~mm} \mathrm{MgCl}_{2}$ (Roche Diagnostics $\mathrm{GmbH}$, Mannheim, Germany). Primers were added to a final concentration of $34 \mu \mathrm{m}$. In each experiment, $1 \mu \mathrm{g}$ of extracted RNA from the cells was reverse transcribed to generate $\mathrm{cDNAs}$ that were then diluted $1: 10$. Finally, $5 \mu \mathrm{l}$ of the diluted cDNA was added to a capillary tube. One positive control, one negative control, and standards were included in each run. The PCR programs started with a preincubation step for activation of the FastStart enzyme, then continued with amplification, and ended with melting curve analysis. The temperature transition rate was $0.1^{\circ} \mathrm{Cs}^{-1}$. The preincubation and amplification programmes for each line were as follows: hENT1, preincubation at $95^{\circ} \mathrm{C}$ for $10 \mathrm{~min}$ and amplification with 40 cycles of $95^{\circ} \mathrm{C}$ for $10 \mathrm{~s}, 60^{\circ} \mathrm{C}$ for $10 \mathrm{~s}$, and $72^{\circ} \mathrm{C}$ for $10 \mathrm{~s}$; $d C K$, preincubation at $95^{\circ} \mathrm{C}$ for $10 \mathrm{~min}$ and amplification with 40 cycles of $95^{\circ} \mathrm{C}$ for $10 \mathrm{~s}, 56^{\circ} \mathrm{C}$ for $10 \mathrm{~s}$, and $72^{\circ} \mathrm{C}$ for $17 \mathrm{~s}$; $R R M 1$, preincubation at $95^{\circ} \mathrm{C}$ for $10 \mathrm{~min}$ and amplification with 40 cycles of $95^{\circ} \mathrm{C}$ for $10 \mathrm{~s}, 58^{\circ} \mathrm{C}$ for $10 \mathrm{~s}$, and $72^{\circ} \mathrm{C}$ for $10 \mathrm{~s}$; $R R M 2$, preincubation at $95^{\circ} \mathrm{C}$ for $10 \mathrm{~min}$ and amplification with 40 cycles of $95^{\circ} \mathrm{C}$ for $10 \mathrm{~s}, 60^{\circ} \mathrm{C}$ for $10 \mathrm{~s}$, and $72^{\circ} \mathrm{C}$ for $6 \mathrm{~s}$; GAPDH, preincubation at $95^{\circ} \mathrm{C}$ for $10 \mathrm{~min}$ and amplification with 40 cycles of $95^{\circ} \mathrm{C}$ for $10 \mathrm{~s}, 55^{\circ} \mathrm{C}$ for $10 \mathrm{~s}$, and $72^{\circ} \mathrm{C}$ for $13 \mathrm{~s}$. All programs were then followed by a heating step at $95^{\circ} \mathrm{C}$ and a cooling step at $65^{\circ} \mathrm{C}$ for $15 \mathrm{~s}$ each. Each experiment

Table I Sequences of primers used in reverse transcription-PCR

\begin{tabular}{lll}
\hline Gene & Forward primer $\left(\mathbf{5}^{\prime} \mathbf{-} \mathbf{3}^{\prime}\right)$ & Reverse primer $\mathbf{( 5}^{\prime} \mathbf{- 3}^{\prime} \mathbf{)}$ \\
\hline hENTI & AAAGGAGAGGAGCCAAGAGC & GGCCCAACCAGTCAAAGATA \\
dCK & CCCGCATCAAGAAAATCTCC & TCATCCAGTCATGCCAGTC \\
RRMI & GGAGGATTGGTGTGCTGT & GCTGCTCTTCCTICCTGTG \\
RRM2 & CCCGCTGTTCTATGGCTTC & CCCAGTCTGCCTTCTTCTTG \\
GAPDH & ATGACCACAGTCCATGCCAT & TTGAAGTCAGAGGAGACCAC
\end{tabular}

Abbreviations: $\mathrm{dCK}=$ deoxycytidine kinase; $\mathrm{GAPDH}=$ glyceraldehyde-3-phosphate dehydrogenase; hENTI = human equilibrative nucleoside transporter-I. 
using quantitative RT-PCR was performed in triplicate. The expression of $h E N T 1, d C K, R R M 1$, and $R R M 2$ mRNA was quantified relative to GAPDH expression.

The Roche software uses the second derivative maximum method to calculate the fractional cycle numbers where the fluorescence rises above background (crossing point, $C_{\mathrm{p}}$ ), that is, the point at which the rate of change of fluorescence is fastest. For the standard curve, $\mathrm{CpS}$ are plotted vs log concentration for the standards. This standard curve is used to estimate the concentration of each sample. The standard curves were saved in a coefficient file that was used by the relative quantification software from Roche to calculate the mRNA levels relative to GAPDH. This program also corrected for the differences in efficiency of the PCR reaction for each target.

\section{Statistical analysis}

Data are expressed as means \pm s.d. Analysis was performed using the Mann-Whitney $U$-test (two-tailed) for nonparametric data. Correlations between nonparametric data were analysed by the Spearman correlation test.

\section{RESULTS}

Development of acquired gemcitabine resistance in
pancreatic cancer cell lines, PCI43, PK1, and KLM1

Resistance to gemcitabine was successfully induced in the PCI43, PK1, and KLM1 cell lines via exposure to stepwise increases in gemcitabine concentration. Gemcitabine-resistant PCI43, PK1, and KLM1 cells were developed by continuous exposure to increasing concentrations of gemcitabine over a period of 6 months, starting with an initial concentration of $3 \mathrm{~nm}$. The resistant cells obtained, PCI43-G4000, PK1-G4000, and KLM1-G4000, were viable in medium containing $4000 \mathrm{~nm}$ gemcitabine. Resistant cells showed no apparent morphologic differences or difference in growth rate compared with the parental cells.

The $\mathrm{IC}_{50}$ values and resistance ratios for parental cells and gemcitabine-resistant cells are listed in Table 2 . The $\mathrm{IC}_{50}$ values of gemcitabine for the parental PCI43, PK1, and KLM1 cells were 350, 160 , and $80 \mathrm{nM}$, respectively. PCI43-G4000, PK1-G4000, and KLM1G4000 cells were 157-, 625-, and 2625-fold less sensitive to gemcitabine than the parental cell lines, respectively.

\section{Analysis of $h E N T 1, d C K, R R M 1$, and $R R M 2$ mRNA expression by real-time light cycler-PCR in different gemcitabine-resistant cell lines}

To evaluate the expression of hENT1, dCK, RRM1, and RRM2 mRNA, real-time light cycler-PCR was performed in a quantitative manner. The mRNA was extracted from each gemcitabine-resistant cell line and analysed via light cycler. Glyceraldehyde-3-phosphate dehydrogenase was used as an internal control. The quantitative data were summarised from the means of the data gathered from

Table $2 \quad I C_{50}$ values of gemcitabine in parental and gemcitabine-resistant cells of PCl43 and PKI cell lines

\begin{tabular}{lrc}
\hline Cell lines & IC $_{\mathbf{5 0}}$ (nM) of GEM & Resistant ratio \\
\hline PCI43 & 350 & \\
PCl43-G4000 & 55000 & 157 \\
PKI & 160 & \\
PKI-G4000 & 100000 & 625 \\
KLMI & 80 & 2625 \\
KLMI-G4000 & 210000 & \\
\hline
\end{tabular}

the three experiments (Figure 1). Real-time light cycler-PCR demonstrated that PCI43-G4000 cells had approximately 4- and 10 -fold increases in the levels of $R R M 1$ and $R R M 2$ mRNA compared with the parental cells, respectively. PK1-G4000 cells had approximately a two-fold increase in levels of $R R M 1 \mathrm{mRNA}$, but there was no increase of RRM2 mRNA compared with parental cells. Similarly, KLM1-G4000 cells had approximately a 42 -fold increase in levels of RRM1 mRNA, but no increase of RRM2 mRNA compared with parental cells. Human equilibrative nucleoside transporter-1 gene expression was significantly increased compared with parental cells in PCI43-G4000 and KLM1-G4000 cells, but not in PK1-G4000 cells. Expression of $d C K$ mRNA was not detected by real-time light cycler-PCR in either PCI43-G4000 or PK1-G4000 cells, and no changes in $d C K$ mRNA expression were observed in KLM1-G4000 cells. To determine the cellular modification responsible for $d C K$ mRNA downregulation, seven exons of the $d C K$ gene as well as the $5^{\prime}$-untranslated regions were amplified by PCR as described previously (Galmarini et al, 2004). In PCR products obtained using genomic DNA from cells, as shown in Figure 2, a partial deletion of the $d C K$ gene was amplified in both PCI43-G4000 and PK1-G4000 cells. No deletion of the $d C K$ gene was detected in parental cell lines or KLM1-G4000 cells.

Gene expression changes in the process of acquired gemcitabine resistance by real-time light cycler-PCR in pancreatic cancer cells

It is not fully understood how gemcitabine influences its own transport and metabolism in the development of gemcitabine resistance. To clarify the changes of gene expression in the process of acquired resistance, we established seven gemcitabine-resistant subclones of the PCI43, PK1, and KLM1 cell lines. Examination of the gemcitabine-resistant PCI43 subclones revealed a proportional
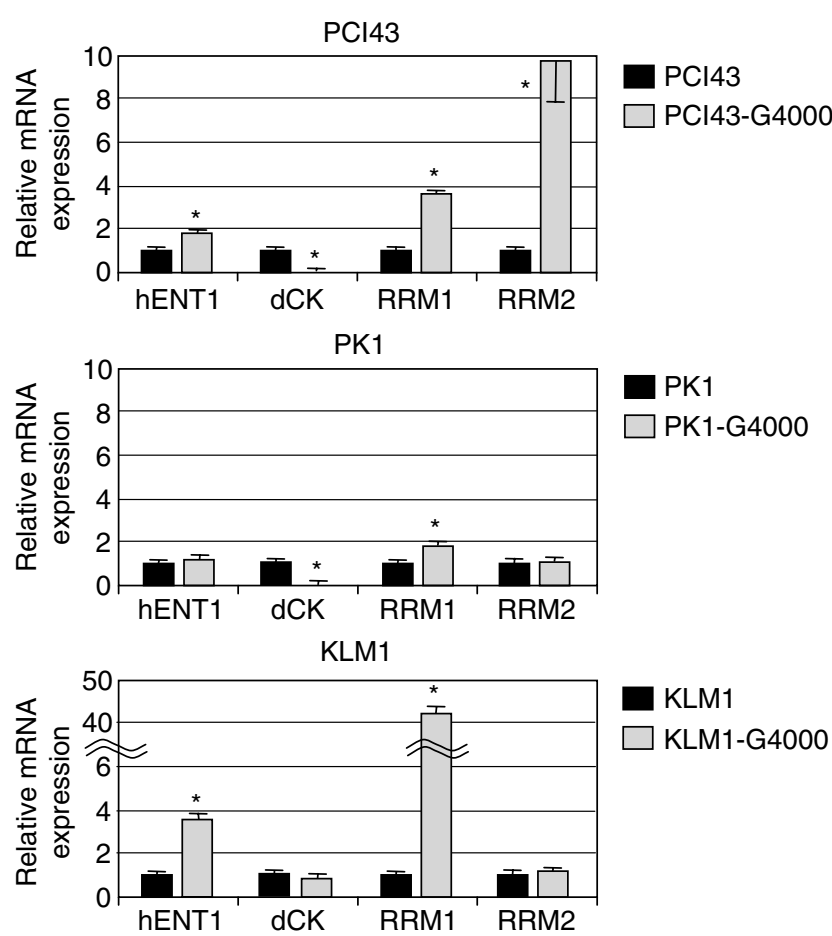

Figure I mRNA expression relative to GAPDH in parental and gemcitabine-resistant cells of PCI43, PKI, and KLMI cell lines, respectively. Each $I C_{50}$ value is the mean of the values in three independent sensitivity tests performed in quadruplicate. Expression levels are relative to expression of GAPDH. ${ }^{* P}<0.05$ to parental cells. Bars, s.d. 
increase in RRM2 message with increasing gemcitabine exposure (Figure 3). RRM1 mRNA showed a two- to four-fold up regulation of expression levels. Significant increases in $R R M 1$ and $R R M 2$ gene expression induced by gemcitabine were detected in the subclones PCI43-G30 and -G3, respectively. No $d C K$ mRNA could be detected in subclones PCI43-G3000 and -G4000, which had higher resistance to gemcitabine, but the $d C K$ gene was slightly increased at lower concentrations of gemcitabine. The hENT1 gene was slightly increased in the latter phase of development of acquired resistance. In the gemcitabine-resistant PK1 subclones, a slight

\section{$\mathrm{PCl} 43$ and $\mathrm{PCl} 43-\mathrm{G} 4000$}

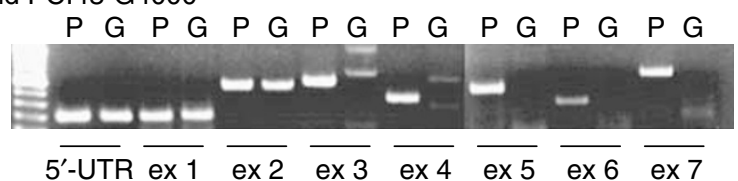

PK1 and PK1-G4000

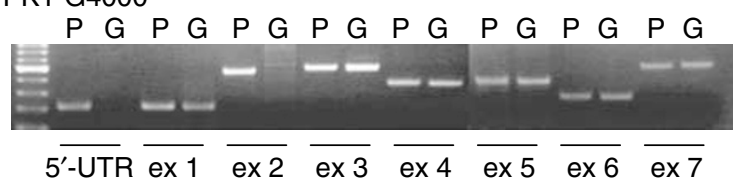

KLM1 and KLM1-G4000

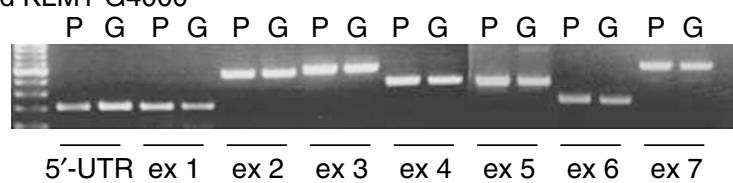

Figure 2 Analysis of dCK PCR product of parental cells and $\mathrm{PCl} 43$ G4000, PKI-G4000, and KLMI-G4000, on agarose gel electrophoresis stained with ethidium bromide. The $5^{\prime}$-untranslated region and complete coding sequence of exons $1-7$ of the human $d C K$ gene were amplified. P: parental cells, G: gemcitabine resistant. increase in RRM1 mRNA was detected, but no increase in the $R R M 2$ gene was observed. No $d C K$ mRNA could be detected in subclones PK1-G300 to -G4000. The gemcitabine-resistant KLM1 subclones showed marked upregulation in $R R M 1 \mathrm{mRNA}$, but there was no increase in the $R R M 2$ gene. $d C K$ mRNA could be detected in all KLM1 subclones. These results suggest that acquired resistance of pancreatic cancer cells to gemcitabine may be determined by the balance of these four factors, but is not predicted by that of a single gene. Decreased hENT1 or $d C K$ has been reported to promote gemcitabine resistance (Ruiz van Haperen et al, 1994; van der Wilt et al, 2000; Garcia-Manteiga et al, 2003; Jordheim et al, 2003; Galmarini et al, 2004). In contrast, increased expression of RR has been reported to be associated with gemcitabine resistance in human tumour cells (Goan et al, 1999; Jung et al, 2001). For these reasons, we calculated the ratio of $h E N T 1 \times d C K / R R M 1 \times R R M 2$ gene expression in gemcitabineresistant subclones. As shown in Figure 4, the ratio of $h E N T 1 \times$ $d C K / R R M 1 \times R R M 2$ expression progressively decreased in the process of acquired gemcitabine resistance.

\section{Correlation of the hENT1 $\times \mathrm{dCK} / \mathrm{RRM} 1 \times \mathrm{RRM} 2$ ratio with gemcitabine chemosensitivity in pancreatic cancer cell lines}

To determine whether the $h E N T 1 \times d C K / R R M 1 \times R R M 2$ ratio correlated with gemcitabine sensitivity, we examined the relative mRNA expression of $h E N T 1, d C K, R R M 1$, and RRM2 to GAPDH in eight human pancreatic cancer cell lines. Correlations of $\mathrm{IC}_{50}$ values and relative levels of gene expression in each cell lines are summarised in Table 3 . We found that the $\mathrm{IC}_{50}$ values of gemcitabine did not significantly correlate with relative expression levels of $h E N T 1, d C K, R R M 1$, or $R R M 2$. Next, the ratio of $h E N T 1 \times d C K / R R M 1 \times R R M 2$ expression was calculated and the correlation with gemcitabine sensitivity was determined (Figure 5). Cells with a higher $h E N T 1 \times d C K / R R M 1 \times R R M 2$ expression ratio showed higher gemcitabine chemosensitivity, whereas cells with a lower ratio showed higher chemoresistance. The

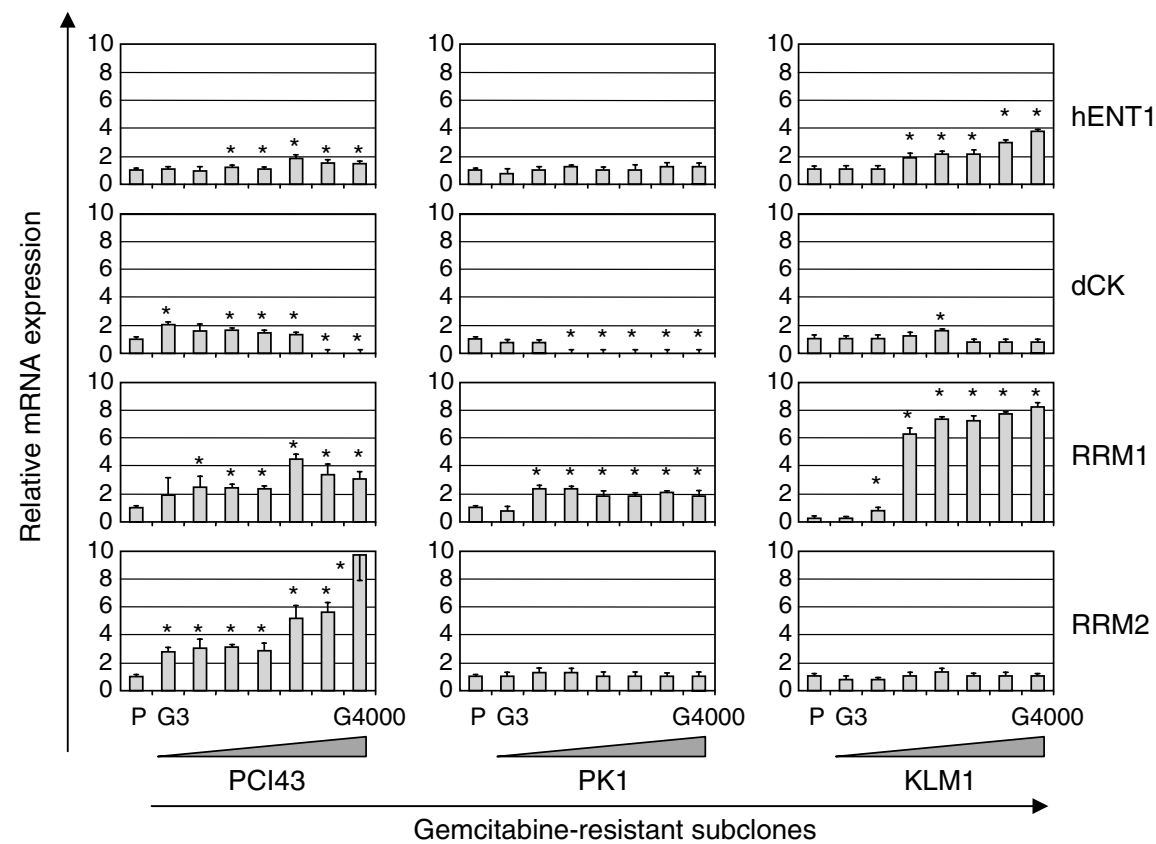

Figure 3 Relationship between gemcitabine resistance and expression levels of hENTI, dCK, RRMI, and RRM2 mRNA in the process of acquired gemcitabine resistance in gemcitabine-resistant PCI43, PKI, and KLMI subclones. Relative mRNA expression to GAPDH was calculated. The value of relative mRNA expression in parental cells was assigned as 1.0. Each point represents the mean \pm s.d. of quadruplicate determinations per plate repeated in triplicate. P: parental cells, G: gemcitabine-resistant. ${ }^{*} P<0.05$ to parental cells. Bars, s.d. 
Table 3 Relative hENTI, dCK, RRMI, and RRM2 mRNA expression to GAPDH in human pancreatic cancer cell lines

\begin{tabular}{lccccc}
\hline & & \multicolumn{4}{c}{ Relative mRNA expression to GAPDH } \\
\cline { 3 - 6 } Cell lines & IC $_{\mathbf{5 0}} \mathbf{( n M )}$ & hENTI & dCK & RRMI & RRM2 \\
\hline PCI43 & 350 & $1.00 \pm 0.12$ & $0.36 \pm 0.0 .3$ & $0.43 \pm 0.02$ & $0.50 \pm 0.04$ \\
PKI & 160 & $1.00 \pm 0.13$ & $1.00 \pm 0.16$ & $1.00 \pm 0.13$ & $1.00 \pm 0.21$ \\
KLMI & 80 & $3.88 \pm 0.22$ & $0.81 \pm 0.02$ & $1.08 \pm 0.04$ & $1.46 \pm 0.13$ \\
PK9 & 70 & $1.12 \pm 0.17$ & $0.99 \pm 0.05$ & $0.31 \pm 0.02$ & $0.63 \pm 0.06$ \\
PK8 & 40 & $3.45 \pm 0.28$ & $2.51 \pm 0.07$ & $0.71 \pm 0.04$ & $1.82 \pm 0.19$ \\
MIAPaCa2 & 40 & $2.64 \pm 0.42$ & $0.42 \pm 0.08$ & $0.42 \pm 0.07$ & $0.47 \pm 0.04$ \\
KPIN & 40 & $23.26 \pm 1.33$ & $2.59 \pm 0.04$ & $1.58 \pm 0.02$ & $4.31 \pm 0.41$ \\
BxPC3 & 30 & $3.52 \pm 0.51$ & $1.20 \pm 0.21$ & $0.70 \pm 0.08$ & $0.84 \pm 0.16$ \\
P-value to IC 50 & & NS & NS & NS & NS \\
\hline NS: not & & & & &
\end{tabular}

NS: not significant. Abbreviations: $\mathrm{dCK}=$ deoxycytidine kinase; $\mathrm{GAPDH}=$ glyceraldehyde-3-phosphate dehydrogenase; hENTI = human equilibrative nucleoside transporter-1.

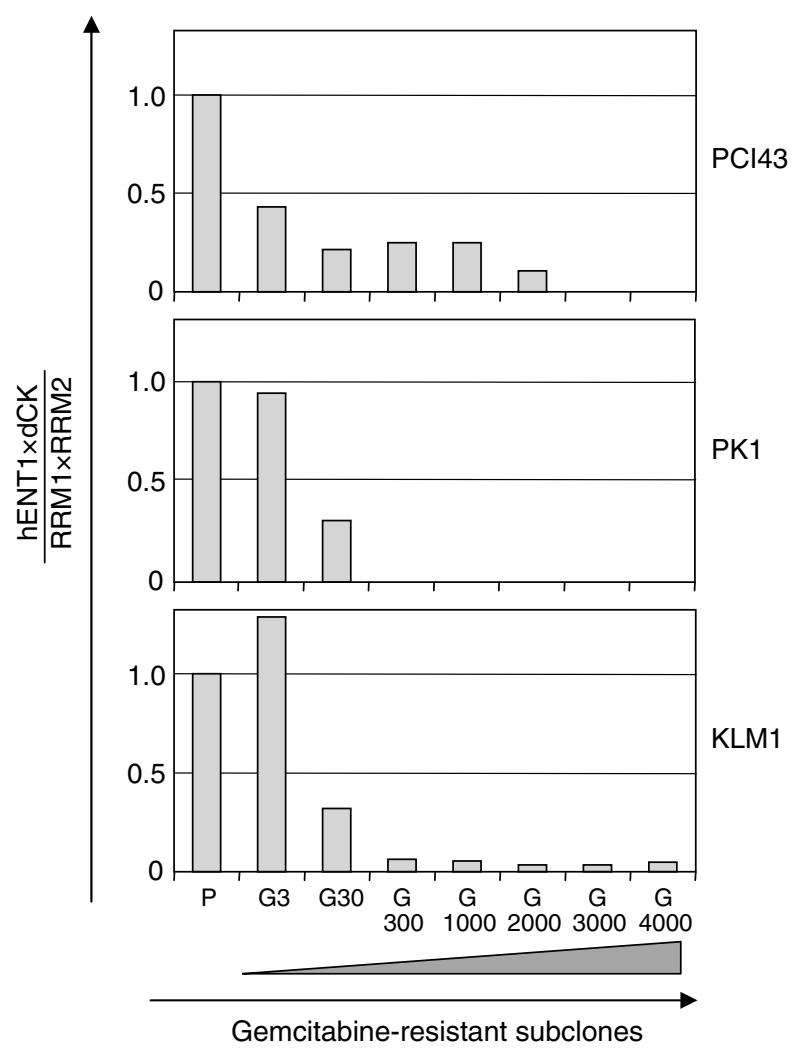

Figure 4 Changes in the $h E N T I \times d C K / R R M I \times R R M 2$ expression ratio in gemcitabine-resistant PCI43, PKI, and KLMI subclones. Value of the ratio in parental cells was set as I.0. The ratio of hENTI $\times d C K / R R M I \times R R M 2$ expression progressively decreased in the process of acquired gemcitabine resistance. P: parental cells, G: gemcitabine resistant.

$h E N T 1 \times d C K / R R M 1 \times R R M 2$ ratio significantly correlated with the gemcitabine $I_{50}$ in eight pancreatic cancer cell lines $(P=0.0029)$. Neither the $R R M 1 \times R R M 2$ ratio, the hENT1/ $R R M 1 \times R R M 2$ ratio, nor the $d C K / R R M 1 \times R R M 2$ ratio correlated with sensitivity to gemcitabine.

\section{DISCUSSION}

Chemoresistance is a major cause of pancreatic adenocarcinoma treatment failure with gemcitabine. The majority of patients with

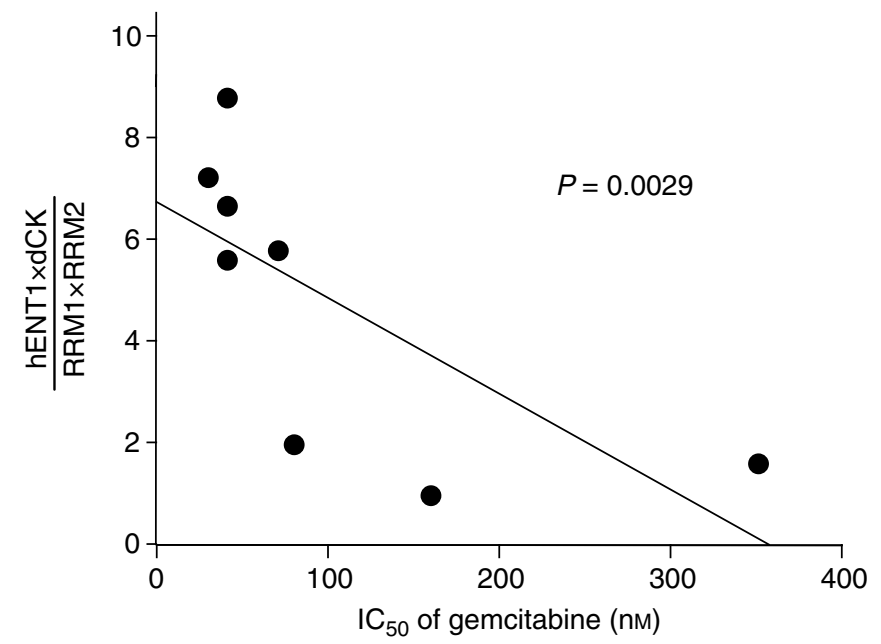

Figure 5 Significant correlation between the $\mathrm{IC}_{50}$ of gemcitabine and the value of $h E N T I \times d C K / R R M I \times R R M 2$ expression ratio in eight pancreatic cancer cell lines $(P=0.0029)$. Cells with a higher $h E N T I \times d C K J$ $R R M I \times R R M 2$ expression ratio showed higher gemcitabine chemosensitivity, whereas cells with a lower ratio showed higher chemoresistance.

gemcitabine-treated pancreatic adenocarcinoma become resistant after consecutive treatments, and fail to derive benefit from chemotherapy. Therefore, it is extremely important to clarify the mechanism behind chemoresistance and to identify predictive markers of inherent and acquired chemoresistance to gemcitabine for better treatment of these tumours.

It has been shown that modulation of cellular enzymes of gemcitabine transport and metabolism influences drug activity in vitro (Mackey et al, 1998, 1999; Goan et al, 1999; Ritzel et al, 2001). Cellular enzymes of gemcitabine transport and metabolism, that is, hENT1, dCK, RRM1, and RRM2, are well documented. Moreover, experimental data may improve the success of pancreatic cancer treatment either by selecting responsive patients or by modulation of gemcitabine effect with rationally selected drug combinations. For these reasons, this study addressed the transcription analysis of hENT1, dCK, RRM1, and RRM2 in parental cell lines and in isolated variable resistant subclones continuously exposed to gemcitabine to determine possible predictive markers for gemcitabine resistance. Our data demonstrate for the first time that acquired and inherent chemoresistance of pancreatic cancer cells to gemcitabine is determined by the balance of $d C K, R R M 1, R R M 2$, and $h E N T 1$ gene expression, but not to that of any of the individual genes. The $h E N T 1 \times d C K / R R M 1 \times R R M 2$ expression ratio significantly correlates with resistance to gemcitabine in pancreatic cancer cells, including acquired gemcitabine-resistant cells, suggesting that a decrease of this ratio reflects inherent and acquired chemoresistance of pancreatic cancer cells to gemcitabine and may be a key to understanding the variable effectiveness of gemcitabine among individual patients. The expression ratio is a novel, informative marker for predicting and monitoring the responses of pancreatic cancer patients to gemcitabine.

A recent study performed on cultured cancer cell lines indicated that hENT1 is the major gemcitabine transporter in human pancreatic cancer cells (Garcia-Manteiga et al, 2003). It is speculated that populations of cells with lower hENT1 abundance may be relatively gemcitabine resistant owing to reduced intracellular accumulation. In fact, pharmacological inhibition of hENT1 in cells has been reported to render them gemcitabine resistant (Mackey et al, 1998). However, the current study shows that expression of the hENT1 gene was not reduced in the development of gemcitabine resistance, and did not correlate with $\mathrm{IC}_{50}$ values of gemcitabine in eight pancreatic cancer cell lines. 
462

These data suggest that $h E N T 1$ expression alone does not reflect inherent and acquired resistance to gemcitabine. The increase in hENT1 expression in the PK1-G4000, PCI-G4000, and KLM1-G4000 subclones may be regarded as a compensatory adaptation to higher chemoresistance to gemcitabine.

Previous reports in cells and animal models cited $d C K$ mutation/ deficiency as the main mechanism for gemcitabine resistance in cells with an acquired resistance (Ruiz van Haperen et al, 1994; van der Wilt et al, 2000; Galmarini et al, 2001; Jordheim et al, 2003). In this study, expression of the $d C K$ gene was slightly increased in subclones that were resistant to lower concentrations of gemcitabine, and was undetectable in subclones with high resistance to gemcitabine, that is, PCI43-G4000 and PK1-G4000. Furthermore, we found that expression of the $d C K$ gene alone does not correlate with sensitivity to gemcitabine in eight pancreatic cancer cell lines. Deficiency of $\mathrm{dCK}$ described in previous reports was mainly based on studies performed on highly gemcitabine-resistant clones, and quite different from the clinical setting. Our data indicate that dCK deficiency is involved in a higher grade of acquired gemcitabine resistance, but not in a lower grade of resistant cells or in parental cells.

Expression of RR has been reported to be one of the determinants of gemcitabine chemoresistance in human tumour cells (Jung et al, 2001). In fact, artificial overexpression of RRM2 results in a further increase in gemcitabine chemoresistance (Goan et al, 1999). An increased level of RR expands the size of the dNTP pools, which competitively inhibits the incorporation of gemcitabine triphosphate into DNA (Plunkett et al, 1996). The expanded dNTP pools further downregulate the activity of dCK via a negative-feedback pathway. Results from this study demonstrate that RRM1 mRNA expression was markedly increased in the KLM1 gemcitabineresistant cells over that of the parental cells. This result supports the previous report that RRM1 is the marker predicting resistance to gemcitabine in lung cancer cell lines (Davidson et al, 2004). In this study, KLM1 gemcitabine-resistant cells had no $d C K$ deficiency in contrast to the lack of $d C K$ in PCI43 and PK1 gemcitabine-resistant cells. Therefore, these data demonstrate that RRM1 may correlate with acquired resistance to gemcitabine, especially in cells without dCK deficiency. RRM2 mRNA was increased in PCI43 gemcitabineresistant cells, but not in PK1 and KLM1 gemcitabine-resistant subclones. In the eight pancreatic carcinoma cells tested, the cells with higher inherent resistance to gemcitabine did not show higher levels of $R R M 1$ and $R R M 2$ expression. These results suggest that an increase in either $R R M 1$ or $R R M 2$ expression alone correlates with acquired chemoresistance, but does not reflect inherent chemoresistance to gemcitabine. In the development of gemcitabine resistance, increases in either RRM1 or RRM2 expression, in addition to a decrease in $d C K$, may indicate that cells are regulated to avoid damage by gemcitabine incorporation in gemcitabineresistant cells.

\section{REFERENCES}

Agarwal B, Abu-Hamda E, Molke KL, Correa AM, Ho L (2004) Endoscopic ultrasound-guided fine needle aspiration and multidetector spiral CT in the diagnosis of pancreatic cancer. Am J Gastroenterol 99: $844-850$

Arnold NB, Ketterer K, Kleeff J, Friess H, Buchler MW, Korc M (2004) Thioredoxin is downstream of Smad7 in a pathway that promotes growth and suppresses cisplatin-induced apoptosis in pancreatic cancer. Cancer Res 64: 3599-3606

Burris III HA, Moore MJ, Andersen J, Green MR, Rothenberg ML, Modiano MR, Cripps MC, Portenoy RK, Storniolo AM, Tarassoff P, Nelson R, Dorr FA, Stephens CD, Von Hoff DD (1997) Improvements in survival and clinical benefit with gemcitabine as first-line therapy for patients with advanced pancreas cancer: a randomized trial. J Clin Oncol 15: $2403-2413$

Carmichael J, Fink U, Russell RC, Spittle MF, Harris AL, Spiessi G, Blatter J (1996) Phase II study of gemcitabine in patients with advanced pancreatic cancer. Br J Cancer 73: $101-105$
Ribonucleotide reductase plays a role as a central enzyme controlling the rate of dNTP synthesis (Plunkett et al, 1996). It has recently been reported that the physiological factor for ribonucleotide reduction is thioredoxin, which is required for the RR reaction in vitro (Koc et al, 2006). In yeast, mutants lacking thioredoxin had significantly lower dNTP levels, supporting the idea that thioredoxin functions as an $\mathrm{RR}$ reductant in vitro. Interestingly, thioredoxin is identified as a gene whose basal expression is increased in pancreatic cancer cells in which Smad7 is commonly overexpressed (Arnold et al, 2004). Thioredoxin is downstream of smad7 in a pathway that acts to promote growth and induce apoptosis resistance in pancreatic cancer cells. Future studies are necessary to investigate whether or not thioredoxin is downregulated in gemcitabine-resistant pancreatic cancer cells.

Previous genomic analysis of pancreatic cancer has been performed exclusively with surgical and autopsy specimens, owing to the difficulty of tissue sampling without surgery. To overcome this difficulty, endoscopic ultrasonography-guided fine-needle aspiration biopsy (EUS-FNAB) is applied to obtain tumour cells as an effective and safe method for tissue diagnosis of pancreatic cancer (Agarwal et al, 2004). More recently, several investigators have demonstrated that genetic analysis using EUS-FNAB specimens is possible for determination of cancer stage or to improve the accuracy of diagnosis (Tada et al, 2002; Pellise et al, 2004). The current study shows that detection and quantitation of hENT1, $d C K, R R M 1$, and RRM2 mRNA can be performed rapidly and reliably using light cycler-PCR with SYBR Green fluorescence. The analytical strategy using light cycler-PCR with EUS-FNAB specimens will enable us to evaluate the chemoresistance of pancreatic cancer before and after gemcitabine treatment in the clinic.

In summary, quantitative RT-PCR analysis showed that the balance of four gene expression levels correlates with inherent and acquired resistance to gemcitabine in pancreatic cancer cells. We propose that resistance of cancer cells to gemcitabine is determined by the ratio of expression of these genes in pancreatic cancer cells. The ratio of gene expression may be a useful marker for predicting and monitoring the efficacy of gemcitabine therapy in pancreatic cancer patients. Further studies are currently underway using pancreatic cancer tissues obtained by EUS-FNAB to elucidate the importance of the expression ratio in gemcitabine therapy for pancreatic cancer patients.

\section{ACKNOWLEDGEMENTS}

This research was supported in part by a Grant-in-Aid for Scientific Research provided by the Ministry of Education, Culture, Sports, Science and Technology, Japan.

Cory JG, Sato A (1983) Regulation of ribonucleotide reductase activity in mammalian cells. Mol Cell Biochem 53-54: 257-266

Davidson JD, Ma L, Flagella M, Geeganage S, Gelbert LM, Slapak CA (2004) An increase in the expression of ribonucleotide reductase large subunit 1 is associated with gemcitabine resistance in non-small cell lung cancer cell lines. Cancer Res 64: $3761-3766$

Fan H, Villegas C, Wright JA (1996) Ribonucleotide reductase R2 component is a novel malignancy determinant that cooperates with activated oncogenes to determine transformation and malignant potential. Proc Natl Acad Sci USA 93: 14036-14040

Galmarini CM, Clarke ML, Jordheim L, Santos CL, Cros E, Mackey JR, Dumontet C (2004) Resistance to gemcitabine in a human follicular lymphoma cell line is due to partial deletion of the deoxycytidine kinase gene. BMC Pharmacol 24: 8

Galmarini CM, Mackey JR, Dumontet C (2001) Nucleoside analogues: mechanisms of drug resistance and reversal strategies. Leukemia 15: $875-890$ 
Garcia-Manteiga J, Molina-Arcas M, Casado FJ, Mazo A, Pastor-Anglada M (2003) Nucleoside transporter profiles in human pancreatic cancer cells: role of hCNT1 in $2^{\prime}, 2^{\prime}$-difluorodeoxycytidine-induced cytotoxicity. Clin Cancer Res 9: $5000-5008$

Goan YG, Zhou B, Hu E, Mi S, Yen Y (1999) Overexpression of ribonucleotide reductase as a mechanism of resistance to 2,2-difluorodeoxycytidine in the human KB cancer cell line. Cancer Res 59: $4204-4207$

Habiro A, Tanno S, Koizumi K, Izawa T, Nakano Y, Osanai M, Mizukami Y, Okumura T, Kohgo Y (2004) Involvement of p38 mitogen-activated protein kinase in gemcitabine-induced apoptosis in human pancreatic cancer cells. Biochem Biophys Res Commun 316: 71-77

Jordheim L, Galmarini CM, Dumontet C (2003) Drug resistance to cytotoxic nucleoside analogues. Curr Drug Targets 4: 443-460

Jung CP, Motwani MV, Schwartz GK (2001) Flavopiridol increases sensitization to gemcitabine in human gastrointestinal cancer cell lines and correlates with down-regulation of ribonucleotide reductase M2 subunit. Clin Cancer Res 7: 2527-2536

Koc A, Mathews CK, Wheeler LJ, Gross MK, Merrill GF (2006) Thioredoxin is required for deoxyribonucleotide pool maintenance during $\mathrm{S}$ phase. J Biol Chem 281: $15058-15063$

Koizumi K, Tanno S, Nakano Y, Habiro A, Izawa T, Mizukami Y, Okumura T, Kohgo Y (2005) Activation of p38 mitogen-activated protein kinase is necessary for gemcitabine-induced cytotoxicity in human pancreatic cancer cells. Anticancer Res 25: $3347-3353$

Li D, Xie K, Wolff R, Abbruzzese JL (2004) Pancreatic cancer. Lancet 363: $1049-1057$

Mackey JR, Mani RS, Selner M, Mowles D, Young JD, Belt JA, Crawford CR, Cass CE (1998) Functional nucleoside transporters are required for gemcitabine influx and manifestation of toxicity in cancer cell lines. Cancer Res 58: 4349-4357

Mackey JR, Yao SY, Smith KM, Karpinski E, Baldwin SA, Cass CE, Young JD (1999) Gemcitabine transport in xenopus oocytes expressing recombinant plasma membrane mammalian nucleoside transporters. J Natl Cancer Inst 91: $1876-1881$

Pellise M, Castells A, Gines A, Agrelo R, Sole M, Castellvi-Bel S, FernandezEsparrach G, Llach J, Esteller M, Bordas JM, Pique JM (2004) Detection of lymph node micrometastases by gene promoter hypermethylation in samples obtained by endosonography-guided fine-needle aspiration biopsy. Clin Cancer Res 10: $4444-4449$

Plunkett W, Huang P, Searcy CE, Gandhi V (1996) Gemcitabine: preclinical pharmacology and mechanisms of action. Semin Oncol 23: 3-15

Rauchwerger DR, Firby PS, Hedley DW, Moore MJ (2000) Equilibrativesensitive nucleoside transporter and its role in gemcitabine sensitivity. Cancer Res 60: 6075-6079

Ritzel MW, Ng AM, Yao SY, Graham K, Loewen SK, Smith KM, Hyde RJ, Karpinski E, Cass CE, Baldwin SA, Young JD (2001) Recent molecular advances in studies of the concentrative $\mathrm{Na}$-dependent nucleoside transporter (CNT) family: identification and characterization of novel human and mouse proteins (hCNT3 and mCNT3) broadly selective for purine and pyrimidine nucleosides (system cib). Mol Membr Biol 18: 65-72 Rothenberg ML, Moore MJ, Cripps MC, Andersen JS, Portenoy RK, Burris III HA, Green MR, Tarassoff PG, Brown TD, Casper ES, Storniolo AM, Von Hoff DD (1996) A phase II trial of gemcitabine in patients with 5FU-refractory pancreas cancer. Ann Oncol 7: 347-353

Ruiz van Haperen VW, Veerman G, Eriksson S, Boven E, Stegmann AP, Hermsen M, Vermorken JB, Pinedo HM, Peters GJ (1994) Development and molecular characterization of a $2^{\prime}, 2^{\prime}$-difluorodeoxycytidine-resistant variant of the human ovarian carcinoma cell line A2780. Cancer Res 54: $4138-4143$

Tada M, Komatsu Y, Kawabe T, Sasahira N, Isayama H, Toda N, Shiratori Y, Omata M (2002) Quantitative analysis of K-ras gene mutation in pancreatic tissue obtained by endoscopic ultrasonography-guided fine needle aspiration: clinical utility for diagnosis of pancreatic tumor. Am J Gastroenterol 97: 2263-2270

Thelander L, Berg P (1986) Isolation and characterization of expressible cDNA clones encoding the M1 and M2 subunits of mouse ribonucleotide reductase. Mol Cell Biol 6: 3433-3442

Van der Wilt CL, Kroep JR, Bergman AM, Loves WJ, Alvarez E, Talianidis I, Eriksson S, Van Groeningen CJ, Pinedo HM, Peters GJ (2000) The role of deoxycytidine kinase in gemcitabine cytotoxicity. Adv Exp Med Biol 486: $287-290$

Zhou BS, Tsai P, Ker R, Tsai J, Ho R, Yu J, Shih J, Yen Y (1998) Overexpression of transfected human ribonucleotide reductase M2 subunit in human cancer cells enhances their invasive potential. Clin Exp Metastasis 16: $43-49$ 